\title{
Modelo SIR de la tendencia pandémica de COVID-19 en Perú
}

\author{
SIR model of the pandemic trend of COVID-19 in Peru
}

Modelo SIR da tendência pandêmica de COVID-19 no Peru

\author{
Ronald Huarachi Olivera ${ }^{1,2}$, Antonio Lazarte Rivera ${ }^{1}$.
}

El trabajo reporta datos desde el inicio de la pandemia de la COVID-19 en Perú aplicado al modelo básico SIR(Susceptibles-Infectados-recuperados) siendo útil para predecir la posibilidad y la gravedad del brote de la enfermedad proporcionando una información clave para determinar el tipo y la intensidad de la intervención de la enfermedad donde se puede evaluar a través de la aparición del pico de número de infectados a fines de Mayo. Por tanto, se deben mantener medidas rigurosas aun cuando las medidas actuales resultan ser efectivas al final de Mayo para evitar una mayor propagación del virus.

\section{Conceptos clave}

Que se sabe sobre el tema:

En base a la efectividad del enfoque de modelado sobre la pandemia debido a la propagación de la nueva enfermedad COVID-19, se desarrolla un modelo Susceptibles-Infecciosos-recuperados o fallecidos (SIR) proporcionando un marco teórico para investigar su propagación dentro de una comunidad.

Que aporta este trabajo:

-Existen pocos estudios que evalúen la caracterización de la evolución epidémica de la enfermedad de coronavirus (COVID-19) en Perú aplicando el modelo SIR (Susceptibles-Infecciososrecuperados o fallecidos).

-Este estudio es el primero en proporcionar una estimación aplicando el modelo SIR de la tendencia pandémica COVID-19, antes y después de que se implementaran las medidas estrictas de prevención y control en el Perú.
1- Universidad Nacional de San Agustin de Arequipa, Perú. Facultad de Ciencias Biológicas. Escuela Profesional de Biología. Laboratorio de Biotecnología Celular y Molecular Avanzada (LABBIOTBEC).

2- E-mail de contacto: ronaldhuarachiolivera@gmail.com

Recibido: 2020-12-02 Aceptado: 2020-12-05

DOI: http://dx.doi.org/10.31053/1853.0605.v78.n3.31142

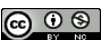

https://creativecommons.org/licenses/by-nc/4.0/

๑) Universidad Nacional de Córdoba

\section{Resumen:}

El virus SARS-CoV-2 procedente de Europa ha llegado a Perú el 5 Marzo y desde el 16 de marzo se ha declarado el estado de emergencia nacional llevando al confinamiento a toda la población. El objetivo de este estudio es caracterizar la evolución epidémica de la enfermedad de coronavirus (COVID-19) aplicando el modelo SIR (SusceptiblesInfecciosos-recuperados o fallecidos) durante un periodo de 200 días. Se utilizó los datos de series temporales de COVID-19 del 06 de marzo al 14 de mayo de 2020 del ministerio de salud peruano planteando casos estimados variando el número básico de reproducción RO. Según el modelo SIR, el pico de infectados se produce poco después del 30 de Mayo desde el inicio de la epidemia (día 86) donde disminuye el número total de casos infectados a $R 0=1,5$. Los resultados sugieren que las estrictas medidas actuales de Perú pueden prevenir eficazmente la propagación de COVID-19 y deben mantenerse aun obteniendo resultados eficientes.

Palabras clave: coronavirus; epidemia; número de reproducción básico.

\section{Abstract:}

The SARS-CoV-2 virus from Europe has reached Peru on March 5 and since March 16 a state of national emergency has been declared, leading to the confinement of the entire population. The objective of this study is to characterize the epidemic evolution of coronavirus disease (COVID-19) applying the SIR model (Susceptible-Infectiousrecovered or deceased) during a period of 200 days. The time series data of COVID-19 from March 06 to May 14, 2020 of the Peruvian Ministry of Health was used, presenting estimated cases by varying the basic reproduction number RO. According to the SIR model, the peak of those infected occurs shortly after May 30 from the beginning of the epidemic (day 86) where the total number of infected cases decreases to R0 $=1.5$. The results suggest that Peru's current stringent measures can effectively prevent the spread of COVID-19 and should be maintained even with efficient results.

Keywords: coronavirus; epidemic; basic reproduction number.

\section{Resumo:}

O vírus SARS-CoV-2 da Europa chegou ao Peru em 5 de março e desde 16 de março foi declarado estado de emergência nacional, levando ao confinamento de toda a população. O objetivo deste estudo é caracterizar a evolução epidêmica da doença coronavírus (COVID-19) aplicando o modelo SIR (Susceptível-Infeccioso-recuperado ou falecido) durante um período de 200 dias. Foram utilizados os dados da série temporal COVID-19 de 06 de março a 14 de maio de 2020 do Ministério da Saúde do Peru, levantando casos estimados variando o número de reprodução básico R0. De acordo com o modelo SIR, o pico de infectados ocorre logo após 30 de maio do início da epidemia (dia 86) onde o número total de casos infectados diminui para R0 $=1,5$. Os resultados sugerem que as atuais medidas rigorosas do Peru podem prevenir efetivamente a disseminação do COVID-19 e devem ser mantidas mesmo com resultados eficientes.

Palavras-chave: coronavírus; epidemia; número básico de reprodução. 


\section{Introducción}

La enfermedad del coronavirus 2019 (COVID-19) comenzó en Wuhan, China a principios de diciembre de 2019, donde se identificaron los primeros casos de una neumonía de origen desconocido en la capital de la provincia de Hubei-China y rápidamente se extendió en ese país y en muchos países y regiones del mundo ${ }^{(1-3)}$. El virus probablemente se originó en un murciélago después de la mutación en la glucoproteína spike, como se sugirió recientemente, adquiriendo la capacidad de infectar a los humanos $^{(4)}$. Los trímeros de glicoproteína de spike(S) de coronavirus promueven la entrada en las células siendo el target principal de la respuesta inmune humoral estableciendo ACE2 como un receptor funcional para este nuevo coronavirus donde los dominios de unión al receptor de SARS-CoV-2 S y SARS-CoV S se unen con afinidades similares a ACE2 humano, lo que se correlaciona con la propagación eficiente de SARS-CoV-2 entre humanos ${ }^{(5)}$.

El surgimiento del coronavirus 2 del síndrome respiratorio agudo severo (SARS-CoV-2); denominado provisionalmente coronavirus de 2019 o enfermedad de 2019-nCoV (COVID-19) en China a fines de 2019, ha causado un gran brote global y es uno de los principales problema de salud pública. A partir del 11 de febrero de 2020, los datos de la Organización Mundial de la Salud (OMS) han demostrado que se han identificado más de 43.000 casos confirmados en 28 países y regiones, con más del $99 \%$ de los casos detectados en China. El 30 de enero de 2020, la OMS declaró a COVID-19 como la sexta emergencia de salud pública de interés internacional. EI SARS-CoV-2 está estrechamente relacionado con dos coronavirus similares al síndrome respiratorio agudo severo derivado del murciélago, bat-SL-CoVZC45 y bat-SLCoVZXC21. Se contagia por transmisión de persona a persona a través de gotitas o contacto directo, y se estima que la infección tiene un período de incubación medio de 6,4 días. Entre los pacientes con neumonía causada por el SARS-CoV-2 (nueva neumonía por coronavirus o neumonía de Wuhan), la fiebre es el síntoma más común, seguido de tos. La afectación pulmonar bilateral con opacidad en vidrio esmerilado fue el hallazgo más común en las imágenes de tomografía computarizada del tórax ${ }^{(6)}$.

El $81 \%$ de los enfermos COVID-19 hace cuadro leve; el $14 \%$, grave; $y$ el $5 \%$ requiere cuidados intensivos ${ }^{(7)}$.

A partir del 11 de febrero de 2020, los datos de la OMS mostraron que había un total de 43.103 casos de COVID-19 (https://www.who.int/docs/default-source/coronaviruse/situation-

reports/20200211-sitrep-22-ncov.pdf?sfvrsn=6f80d1b9 4). Se confirmó que la pandemia de COVID-19 llegó a América Latina en febrero de 2020 con una expansión gradual en la región hasta marzo de 2020, cuando la curva de incidencia de COVID-19 comenzó a crecer más rápidamente. En América Latina, hasta el 27 de Julio de 2020 Brasil ha reportado 2.423 .798 casos, el mayor número de casos en la región seguido por Perú con un total de $384.797 \operatorname{casos}^{(8)}$.

El Perú, es un país ubicado en el oeste de Sudamérica, informó su primer caso importado de COVID-19 en Lima, el 6 de marzo de 2020, un peruano con un historial reciente de viajes a Francia, España y República Checa ${ }^{(9-10)}$. Para el 15 de abril de 2020, el gobierno peruano había informado un total de 11.475 casos, incluidas 254 muertes. Lima, la capital del Perú ha registrado 8.412 casos, el mayor número de casos en Perú(11). Para responder al creciente número de casos de COVID-19 en el país, el gobierno cerró las escuelas el 11 de marzo de 2020. Al día siguiente, el gobierno prohibió las reuniones de más de 300 personas y suspendió todos los vuelos internacionales desde Europa y Asia. El 16 de marzo de 2020, el gobierno peruano declaró emergencia nacional y cierre de fronteras en el país ${ }^{(12)}$. Posteriormente, el $17 \mathrm{de}$ marzo de 2020, el presidente de Perú anunció el comienzo de la transmisión comunitaria del SARS-CoV-2 en el país y ordenó el toque de queda en la región el 18 de marzo de 2020 para evitar la socialización nocturna para prevenir la transmisión de enfermedades. Hasta el 12 de Mayo 2020 en Perú, se han registrado 820 médicos contagiados con el nuevo coronavirus
SARS CoV-2 (COVID-19), donde 16 médicos fallecieron a causa de esa enfermedad ${ }^{(12-13)}$.

Hasta el 12 de mayo de 2020, 212 países habían confirmado 4.335.709 casos y 292.291 fallecimientos. Los datos de la organización Mundial de la salud (OMS) indican que los países con mayor cantidad de casos son Estados Unidos (1.407.284), España (269.520), Rusia (232.243), UK (226.463), Italia (221.216). Los países con mayor mortalidad hasta esta fecha, son Estados Unidos (83 301), Reino Unido (32.692), Italia (30.911), Francia (26.991), España (26.920) y Brasil (12.400) ${ }^{(8)}$.

En Perú, hasta el 14 de Mayo de 2020 se han notificado 80.604 casos, de los cuales 2267 pacientes habían muerto (letalidad $=2,81 \%$ ) y 25.151 pacientes se habían recuperado (tasa de recuperación=31,2\%). En Lima se concentraba el $65,2 \%$ de los casos, seguido de los distritos del Callao, Lambayeque, Piura y Loreto $^{(11)}$.

El modelo matemático es útil para predecir la posibilidad y la gravedad del brote de la enfermedad y proporcionar una información clave para determinar el tipo y la intensidad de la intervención de la enfermedad. El ritmo reproductivo básico (R0), un parámetro que aproxima el promedio de casos nuevos generados por un paciente COVID-19 durante su periodo infeccioso, varía entre 1,4 y 2,5 ; donde $R 0$ es una indicación de la transmisibilidad de un virus, que representa el número promedio de nuevas infecciones generadas por una persona infecciosa en una población totalmente ingenua. Para $\mathrm{RO}>1$, es probable que el número de infectados aumente, y para $\mathrm{R} 0<1$, es probable que la transmisión se extinga. El número básico de reproducción es un concepto central en la epidemiología de las enfermedades infecciosas, que indica el riesgo de un agente infeccioso con respecto a la propagación de la epidemia ${ }^{(14)}$. Dentro de la epidemiología, uno de los modelos matemáticos determinísticos más usado es el denominado modelo SIR (Susceptible-Infectious-Recovered). Este modelo y sus modificaciones, se ha aplicado ampliamente al brote actual de COVID-19. Manrique-Abril, et al. ${ }^{(15)}$ estimaron la infectividad de COVID-19 en base a un modelo epidemiológico clásico (SIR) susceptible-expuesto-infectado. Además, la toma de decisiones de salud pública para controlar una epidemia a menudo se traduce en la reducción de R0; esto se puede hacer de muchas maneras, como la reducción de $\beta$ a través de mejores medidas de protección, como el lavado de manos y el uso de protectores faciales, en el caso de la gripe $^{(16)}$. Si bien la noción de Ro ha dado muchas ideas sobre la propagación de epidemias, tiene varias limitaciones, especialmente en la práctica ${ }^{(17)}$.

Se adoptó el modelo SIR, que cubre los efectos de diferentes medidas de prevención de epidemias como el confinamiento, el distanciamiento y la adopción de medidas de higiene y protección por parte de la población en diferentes períodos con el objetivo de caracterizar la evolución epidémica y predecir la tendencia pandémica de COVID-19 en Perú a través de un modelo SIR.

\section{Material y Métodos}

\section{Fuente de datos}

Con una población total de 32.131 .400 habitantes en Perú (18), se realizó un modelo SIR a la situación de la crisis sanitaria por la COVID-19, se han utilizado los datos diarios disponibles sobre el número de infectados por el COVID-19 en Perú, procedentes del Ministerio de Salud de Perú. En el momento de la extracción, la serie de datos está disponible desde el 06-03-2020 hasta el 14-052020.

Se utilizó un modelo SIR con enfoque determinístico para pronosticar el desarrollo de la pandemia de COVID-19 en Perú. Los estados considerados fueron susceptibles (S), infecciosos (I) y fallecidos (R). Los cálculos se realizaron con los programas Excel囚 versión 2008

\section{Preparación del modelo}

El modelo de epidemia SIR (Susceptible-Infectious-Recovered) considera la evolución temporal de una población susceptible, que 
interactúa con una población infectada. El modelo SIR describe la dinámica del sistema: Cada persona infectada puede infectar a cualquier persona susceptible (independientemente) con probabilidad $\beta$ y puede recuperarse con probabilidad $\gamma$. Dado que $\mathrm{S}(\mathrm{t})$, I(t) y $\mathrm{R}(\mathrm{t})$ denotan el número de personas susceptibles, infectadas y recuperadas en el momento $t$, respectivamente. Sea $\mathrm{s}(\mathrm{t})=\mathrm{S}(\mathrm{t}) / \mathrm{N}, \mathrm{i}(\mathrm{t})=\mathrm{I}(\mathrm{t}) / \mathrm{N}, \mathrm{yr}(\mathrm{t})=\mathrm{R}(\mathrm{t}) / \mathrm{N}$; entonces, $\mathrm{s}(\mathrm{t})+\mathrm{i}(\mathrm{t})+\mathrm{r}(\mathrm{t})=1$. Bajo el supuesto de "mezcla completa" de que cada individuo está en contacto con todos en la población, la dinámica del modelo SIR puede ser descrita por un sistema de ecuaciones diferenciales ${ }^{(17)}$.

Uno de los resultados clásicos en el modelo SIR es que hay una epidemia que infecta a una gran fracción de la población, si y solo si $R 0=\beta / y>1$. Al comienzo de una epidemia, gran parte del esfuerzo de salud pública se centra en estimar R0 a partir de infecciones observadas ${ }^{(19)}$. En base al número de casos diarios de infectados con la COVID-19 en Perú, se estimó la velocidad de transmisión del virus, donde R-naught $(\mathrm{R} 0)$ se refiere al promedio de personas a las que una persona infectada transmite el virus en una población sin inmunidad previa (la variación depende de la edad de la población y sus costumbres sociales) ${ }^{(20)}$.

Se construyó una propuesta de predicción de casos basados en inferencia a través de la ecuación logarítmica y de la regresión lineal, tomando como base los primeros 14 días de la pandemia en Perú, los supuestos del modelo predictivo SIR. Se consideró como primera etapa al período comprendido entre el inicio de la epidemia en Perú con el primer caso reportado (06 de marzo de 2020) hasta el día 14 (20 de marzo). El comportamiento del número de infectados, al inicio, se estimó por medio de una función exponencial de la forma $f(t)=e^{\lambda t}$. El valor de $\lambda$, exponente de la función $I(t)$, se calculó para cada etapa y cada estado. Se numeraron los días de la epidemia, representados por la variable $t$, y se obtuvo el logaritmo natural del número de infectados confirmados correspondientes al número de día. De esta manera, se realizó la linealización de la función exponencial y fue posible calcular la pendiente de la recta por medio de regresión lineal ${ }^{(15)}$.

Se probó el modelo previsto comparando los casos esperados versus los casos observados y se diseñó una serie de tiempo que permitía ver el modelo frente al comportamiento real de la pandemia COVID-19 en Perú.

\section{Resultados}

Desarrollo de la epidemia de COVID-19 en Perú comparado con cuatro diferentes Ro

Se muestra los casos observados en Perú desde el 6 de Marzo hasta el 15 de Mayo del 2020, se realizó el modelo SIR con cuatro diferentes $R 0(1,5,2,2,5$ y 3$)$ (figura 1). Como resultado, se observa que el mejor ajuste de la presentación de casos en Perú con $\mathrm{R} 0>1,5$ hasta el 19 de Marzo (figuras 1 y 2 ).

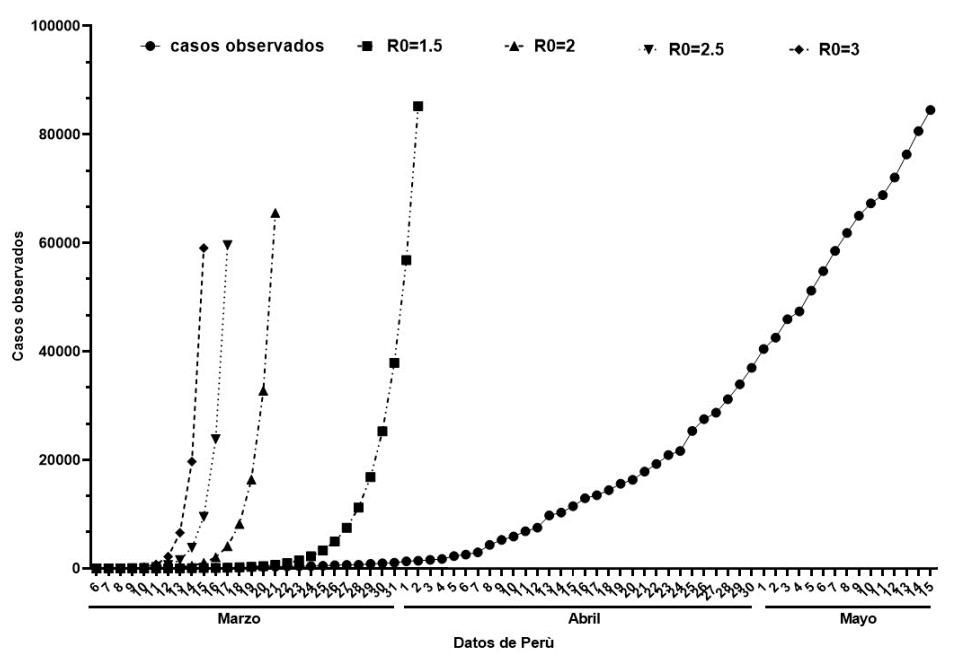

Figura N¹. Casos diarios reportados y esperados según R0 de la pandemia COVID-19 para Perú

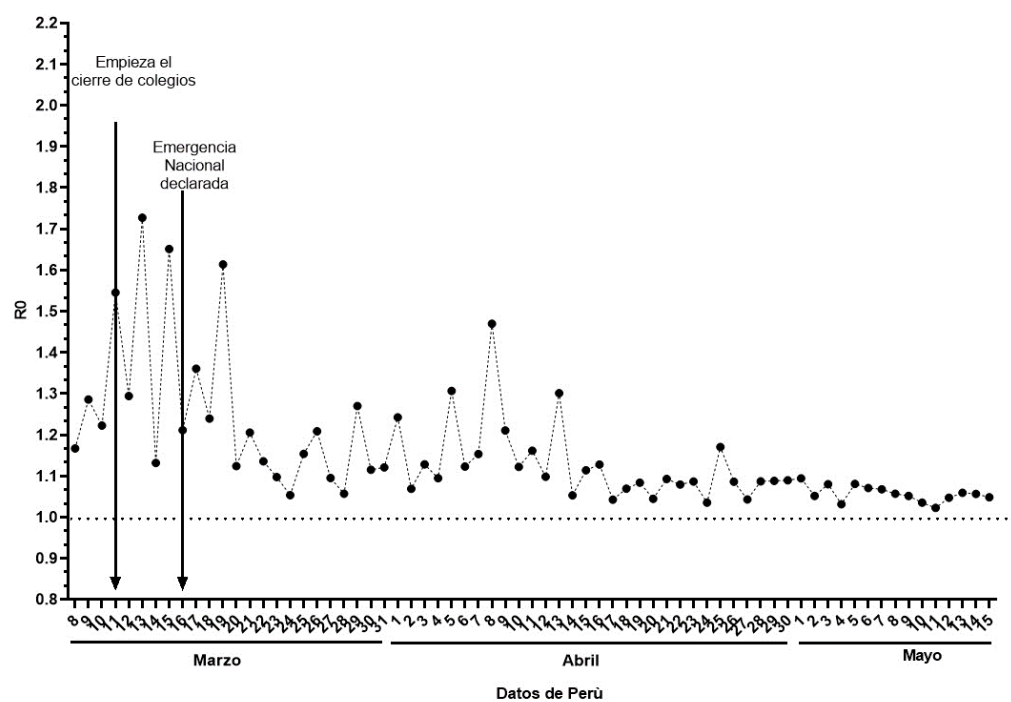

Figura №2. Representación gráfica de R0 para cada período de tiempo. Onda pandémica COVID-19; Perú 06/03/2020-15/05/2020. 
Se observa la estimación del valor de la evolución del número de reproducción básico $\mathrm{R} 0$ con el tiempo, donde el valor de R0 disminuye con el tiempo, con valores acercándose a 1 para el período a partir del 9 de Mayo de 2020, lo que implica que la situación puede estar bajo control en el Perú si es que baja a R0 < 1 (figura 2).
Predicción de la tendencia pandémica de COVID-19 en Perú Se ha simulado la evolución de la pandemia COVID-19 en Perú con una población total de 32.131 .400 de habitantes según el INEI (2020), y en donde el número inicial de infectados al inicio es de una persona. Asimismo, (figuras 3,4 y 5 ).

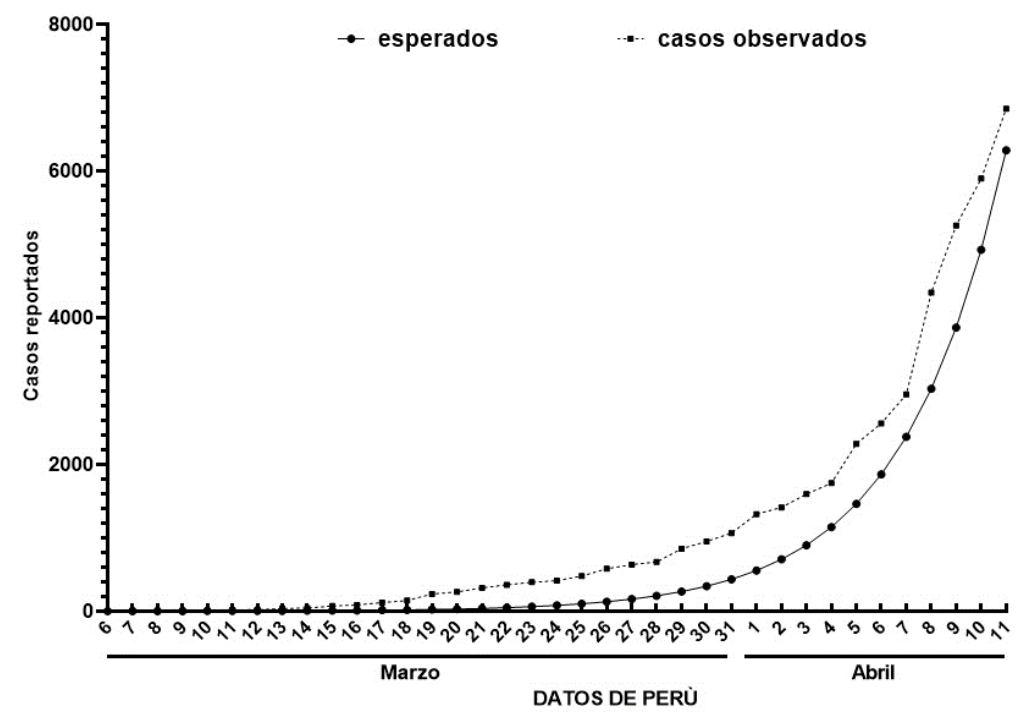

Figura $N^{\circ}$. Casos acumulados reportados y esperados de la pandemia COVID-19 en Perú.

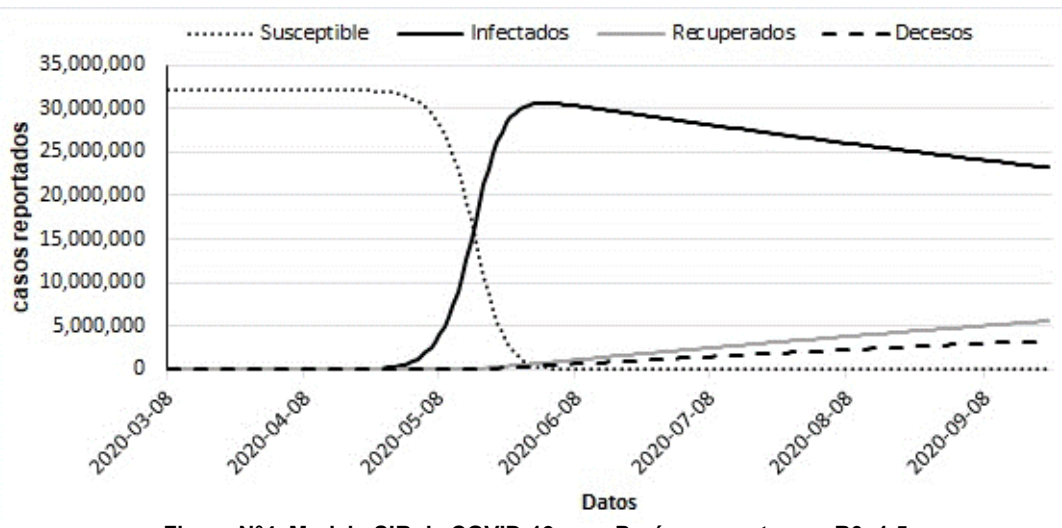

Figura N4. Modelo SIR de COVID-19 para Perú propuesto con R0=1,5.

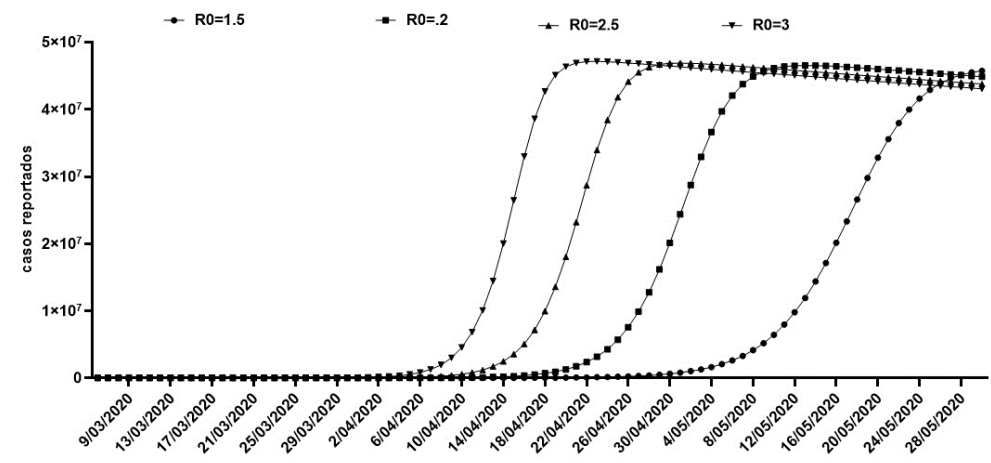

Figura $\mathrm{N}^{\circ} 5$. Proyecciones de contagios modelado hasta el 30 de mayo de 2020 esperados para CoVID-19 en Perú.

Se muestra la evolución con el se ha supuesto una tasa básica de reproducción $(R 0=1,5)$. Se fijó el número de casos esperado por día, y el observado, siguiendo la tendencia mostrada en la ecuación para un $\mathrm{R} 0=1,5$ modelo $\mathrm{SIR}$ a $\mathrm{R} 0=1,5$ durante un periodo de 200 días en el número de individuos susceptibles, infectados y recuperados (figura 4). Como puede apreciarse, el pico de infectados se produce poco después del 30 de Mayo desde el inicio de la epidemia (día 86), en el cuál se registran un total de 30.630.913 nuevos infectados.

Ahora bien, si se mantiene las mismas condiciones del brote bajo los supuestos del modelo, donde se estimó en cuatro fechas futuras los casos para Perú: 2 y 27 de Junio, 17 de Julio y 14 de Agosto descritos en la tabla 1. 
Tabla $\mathbf{N}^{\circ} 1$ : Estimativos del modelo para cuatro fechas futuras-datos 2020

\begin{tabular}{lcccc}
\hline \multicolumn{1}{c}{ Indicadores Días tras el inicio } & 2-jun & 27-jun & 17-jul & 14-ago \\
\hline Población total contagiada & 31.977 .957 & 32.131 .330 & 32.131 .401 & 32.131 .401 \\
Población total recuperada & 854,892 & 2.026 .837 & 2.913 .397 & 4.081 .930 \\
Población total fallecida & 512,935 & 1.216 .102 & 1.748 .038 & 2.449 .158 \\
Población no afectada & 153,444 & 71 & 0 & 0 \\
Máx. población infectada & 30.630 .869 & 30.630 .869 & 30.630 .869 & 30.630 .869 \\
simultánea & 2.197 .806 & 2.197 .806 & 2.197 .806 & 2.197 .806 \\
Máx. contagios diarios & 48,134 & 48,134 & 48,134 & 48,134 \\
Máx. recuperaciones diarias & 28,881 & 28,881 & 28,881 & 28,881 \\
Máx. fallecimientos & & & & \\
\hline
\end{tabular}

\section{Discusión}

El impacto de la respuesta COVID-19 como las regulaciones generales de cuarentena, distanciamiento social y aislamiento de infecciones en China es alentador para muchos otros países ${ }^{(21)}$. Por tanto, los modelos matemáticos han demostrado su efectividad en el apoyo a la toma de decisiones en el control de enfermedades ${ }^{(22)}$. En este trabajo se realizó el modelamiento SIR de la pandemia COVID-19 en Perú con R0 $=1,5$ para predecir en fechas futuras la evolución del número de casos. La propagación de COVID-19 en Perú es preocupante siendo el segundo país de Sudamérica con el mayor número de infectados con la COVID-19 entrando hasta el 15 de Mayo en una fase sin inflexiones, sin lograr achatar la curva del número de infectados, lo que ayuda a observar todo el curso de la epidemia. Además, con respecto a la similitud del tamaño de la población de Perú en comparación con Argentina, Colombia y México donde se registra un mayor tamaño poblacional, el número de infectados COVID-19 es menor. Por tanto, las medidas de salud pública de otros países pueden proporcionar una guía útil para Perú en la prevención de una mayor propagación de COVID-19. Acorde al comportamiento de la pandemia en los diversos países, en Perú se han adelantado medidas para mitigar y frenar su impacto. En especial se busca frenar su velocidad de transmisión con el fin de preparar el sistema de salud para la atención de casos, evitando el colapso y disminuyendo el impacto social y económico de la pandemia. Sin embargo, el Perú tiene los puntajes promedio de 55 en el Informe Anual de Autoevaluación (IAA) y 49 en el Índice de Seguridad Sanitaria Global (GHSI). Para el IAA ocupa la posición 108 entre 183 países, mientras que en el GHSI ocupa el puesto 49 entre 195 países en cuanto a capacidades sobre seguridad sanitaria. Esto ubica al Perú como medianamente preparado para hacer frente a epidemias y pandemias. Respecto a elementos o indicadores específicos sobre capacidades, los puntajes medidos por el GHSI muestran una baja preparación en bioseguridad, sistemas de laboratorios, fuerza laboral en epidemiología, operaciones de respuesta a emergencias, evaluaciones externas conjuntas (JEE, por sus siglas en inglés) y en el desempeño de servicios veterinarios (PVS, por sus siglas en inglés) ${ }^{(23)}$. La epidemia de COVID-19 en Lima siguió una tendencia de crecimiento exponencial temprana, que se desaceleró y se convirtió en una tendencia de crecimiento casi lineal, que parece estar vinculada a intervenciones de distanciamiento social a gran escala implementadas por el gobierno. Si bien las intervenciones parecen haber disminuido la tasa de transmisión, el número de nuevos casos de COVID-19 continúa acumulándose, lo que destaca la necesidad de continuar el distanciamiento social y los esfuerzos de búsqueda activa de casos para mitigar la transmisión de enfermedades en la región ${ }^{(24)}$.

En este estudio, el modelo SIR se utilizó para evaluar la evolución de la epidemia peruana de COVID-19. En estudios anteriores, la estimación de la epidemia de una enfermedad infecciosa a menudo se realiza utilizando el parámetro constante. La desventaja del modelo SIR a diferencia del eSIR extendido no es posible combinar medidas de aislamiento variables en el tiempo para adaptarse a la tasa de transmisión variable en el tiempo de la población. La pandemia COVID-19 fuera de Lima en Perú ha sido menos severo a excepción de los departamentos de Piura, Lambayeque, Arequipa y Loreto $^{(11)}$.

Este estudio mostró que la COVID-19 se extendió rápidamente por todo el Perù después del 7 de Abril de 2020. Las posibles razones para un crecimiento tan rápido de las infecciones incluyen:

- No se tomaron precauciones más oportunas y no se tomaron medidas preventivas.

- El número de infecciones podría estar infravalorado debido a diagnósticos insuficientes, dado los casos subclínicos o asintomáticos.

Se cree que el período de incubación de COVID-19 es de 14 días después de la exposición, y la mayoría de los casos ocurren aproximadamente de cuatro a cinco días después de la exposición $(1,25,26)$. Por lo tanto, parece imposible mantener totalmente dos o tres casos durante el 6 de Marzo al 12 de Mayo en Perú. Además, el rápido aumento en el número de infecciones después del 7 de Abril podría reflejar una comprensión tardía de la propagación de la COVID-19. Los estudios anteriores han demostrado que las políticas de control gubernamental más rigurosas como la detección temprana, el lavado de manos, el autoaislamiento y la cuarentena doméstica probablemente serán más efectivos que las restricciones de viaje, para mitigar esta pandemia ${ }^{(27)}$ asociado a un aumento más lento de la población infectada así como los esfuerzos de colaboración para combatir el nuevo coronavirus, enfocado a intervenciones domésticas estrictas persistentes y en la vigilancia contra los casos importados exógenos ${ }^{(28)}$, donde las medidas estrictas deben implementarse continuamente así como las necesarias medidas estrictas de salud pública que deben aplicarse en otros lugares del país con un alto número de casos de COVID19 , para reducir R0 a un nivel ideal y controlar la infección ${ }^{(29)}$. Este estudio demuestra que las medidas rigurosas pueden prevenir efectivamente la mayor propagación de COVID-19 en Perú. Según nuestro modelo, Perú aún debe mantener todos los niveles de cuarentenas manteniendo R0 debajo de 1,5 hasta llegar a debajo de 1 tratando de achatar la curva de casos infectados COVID-19. Además, según la experiencia de China, diversas medidas de control, incluida la detección temprana y el aislamiento de personas con síntomas, restricciones de tráfico, asistencia médica, el seguimiento y la detección de entrada o salida pueden evitar la propagación de COVID-19. EI Perú actualmente está experimentando un desafío sin precedentes, gestionando y conteniendo un brote de una nueva enfermedad por coronavirus conocida como COVID-19, hasta el 15 de Mayo de 2020 ocupa el puesto número 12 en el número de contagiados a nivel mundial por encima de China donde el brote comenzó, parece haber sido capaz 
de contener el crecimiento de la epidemia. No obstante, las autoridades deben tomar medidas e implementar medidas de contención. La mejor estrategia es la pronta detección y aislamiento de individuos con síntomas, seguido de intervenciones y recomendaciones públicas dirigidas a reducir la transmisibilidad de la enfermedad; aunque no es eficaz para la erradicar la enfermedad, produciría como efecto de segundo orden un retraso de varios días en el aumento del número de casos infectados ${ }^{(30)}$. Pero la estrategia más efectiva aún necesita ser confirmada por otros estudios. En consecuencia, es necesario aplicar medidas estrictas de salud pública en otros países con un alto número de casos de COVID-19. Este estudio tiene algunas limitaciones basándose en el supuesto de que se han tomado medidas rigurosas como China, España e Italia, aunque este estudio utiliza el modelo SIR para caracterizar la evolución de la epidemia, que es instructivo para la prevención y el control de la epidemia en Perú. En conclusión este estudio es el primero en proporcionar una estimación aplicando el modelo SIR de la tendencia pandémica COVID-19, antes y después de que se implementaran las medidas estrictas de prevención y control en el Perú. Si las medidas actuales de prevención y control son suficientes o no, se puede evaluar a través de la aparición del pico de número de infectados a fines de Mayo. Por tanto, se deben mantener medidas rigurosas aun cuando las medidas actuales resultan ser efectivas al final de Mayo para evitar una mayor propagación del virus.

\section{Limitaciones de responsabilidad:}

La responsabilidad de este trabajo es exclusivamente de los autores.

Conflicto de interés:

Ninguno.

\section{Fuentes de apoyo:}

La presente investigación no contó con fuentes de financiación.

\section{Originalidad:}

Este artículo es original y no ha sido enviado para su publicación a otro medio de difusión científica en forma completa ni parcialmente.

\section{Cesión de derechos:}

Los participantes de este trabajo ceden el derecho de autor a la Universidad Nacional de Córdoba para publicar en la RFCM y realizar las traducciones necesarias al idioma inglés.

\section{Contribución de los autores:}

H-O: Planificación de la investigación, búsqueda de la información, análisis e interpretación de los resultados finales, diseño del análisis estadístico, interpretación de los resultados

L-R: Revisión crítica del manuscrito, gestión de la base de datos y apoyo en la elaboración de gráficos y tablas, apoyo en el proceso metodológico y análisis e interpretación de los resultados.

\section{Bibliografía}

1. Li Q, Guan $X$, Wu P, Wang $X$, Zhou L, Tong $Y$, Ren R, Leung KSM, Lau EHY, Wong JY, Xing X, Xiang N, Wu Y, Li C, Chen Q, Li $D$, Liu $T$, Zhao J, Liu $M$, Tu $W$, Chen $C$, Jin $L$, Yang $R$, Wang $Q$, Zhou S, Wang R, Liu H, Luo Y, Liu Y, Shao G, Li H, Tao Z, Yang Y, Deng Z, Liu B, Ma Z, Zhang Y, Shi G, Lam TTY, Wu JT, Gao GF, Cowling BJ, Yang B, Leung GM, Feng Z. Early Transmission Dynamics in Wuhan, China, of Novel Coronavirus-Infected Pneumonia. N Engl J Med. 2020 Mar 26;382(13):1199-1207. doi: 10.1056/NEJMoa2001316.

2. Benvenuto $D$, Giovanetti $M$, Salemi $M$, Prosperi $M$, De Flora $C$, Junior Alcantara LC, Angeletti S, Ciccozzi M. The global spread of 2019-nCoV: a molecular evolutionary analysis. Pathog Glob Health. 2020 Mar;114(2):64-67. doi: 10.1080/20477724.2020.1725339.

3. Liao $X$, Wang B, Kang $Y$. Novel coronavirus infection during the 2019-2020 epidemic: preparing intensive care units-the experience in Sichuan Province, China. Intensive Care Med. 2020 Feb;46(2):357-360. doi: 10.1007/s00134-020-05954-2.

4. Benvenuto D, Giovanetti M, Ciccozzi A, Spoto S, Angeletti S, Ciccozzi M. The 2019-new coronavirus epidemic: Evidence for virus evolution. J Med Virol. 2020 Apr;92(4):455-459. doi: 10.1002/jmv. 25688.

5. Walls AC, Park YJ, Tortorici MA, Wall A, McGuire AT, Veesler $D$. Structure, Function, and Antigenicity of the SARS-CoV-2 Spike Glycoprotein. Cell. 2020 Apr 16;181(2):281-292.e6. doi: 10.1016/j.cell.2020.02.058.

6. Lai CC, Shih TP, Ko WC, Tang HJ, Hsueh PR. Severe acute respiratory syndrome coronavirus 2 (SARS-CoV-2) and coronavirus disease-2019 (COVID-19): The epidemic and the challenges. Int $J$ Antimicrob Agents. 2020 Mar;55(3):105924. doi: 10.1016/j.jiantimicag.2020.105924.

7. Accinelli RA, Xu CMZ, Yachachin-Chávez JM, Cáceres-Pizarro $J A$, Tafur-Bances KB, Flores-Tejada RG, Paiva Andrade AdC. COVID-19: La pandemia por el nuevo virus SARS-CoV-2. Rev Peru Med Exp Salud Publica. 2020; 37(2). doi: 10.17843/rpmesp.2020.372.5411.

8. Worldometer. COVID-19 coronavirus/cases 2020. Fecha de consulta: 27 de Julio de 2020. Disponible en: https://www.worldometers.info/coronavirus/

9. Aquino M, Garrison C. Peru records first confirmed case of coronavirus, President Vizcarra says. Rueters. 2020 Mar. Disponible en: https://www.reuters.com/article/ushealth-coronavirus-peru/perurecords-first-confirmed-case-of-coronavirus-presidentvizcarra-saysidUSKBN20T1S9

10. Ministerio de Salud. Alerta epidemiológica ante el incremento de casos de COVID-19 en el Perú. 2020 May 13. Disponible en https://cdn.www.gob.pe/uploads/document/file/568926/Alerta 0122020.pdf

11. Ministerio de Salud. Perú. Sala Situacional COVID-19 Perú. $2020 . \quad$ Disponible en: https://covid19.minsa.gob.pe/sala situacional.asp

12. Explorer, A. Coronavirus in Peru- the latest updates. 2020 Apr 6. Disponible en: https://amazonas-explorer.com/is-there-coronavirusin-peru/

13. Writing, M. Coronavirus in Peru: Government decrees mandatory social isolation for 15 days to combat coronavirus. Andina. 2020 Mar 15. Disponible en: https://andina.pe/ingles/noticiaperu-government-decrees-mandatory-social-isolation-for-15-days-tocombat-coronavirus-788411.aspx

14. Liu Y, Gayle AA, Wilder-Smith A, Rocklöv J. The reproductive number of COVID-19 is higher compared to SARS coronavirus. $J$ Travel Med. 2020 Mar 13;27(2):taaa021. doi: 10.1093/jtm/taaa021.

15. Manrique AF, González-Chordá, VM, Gutiérrez Lesmes OA, Tellez Piñerez CF, Herrera-Amaya, GM. Modelo SIR de la pandemia de Covid-19 en Colombia. Rev Salud Publica, 2020; 22, 1-9. doi: 10.15446/rsap.V22.85977.

16. Dimitrov NB, Meyers LA. Mathematical approaches to infectious disease prediction and control. Tutorials in Operations Research. Informrs. 2010; 7, pp. 1-25. doi: 10.1287/educ.1100.0075.

17. Pyne S, Vullikanti AKS, Marathe MV. Big Data Applications in Health Sciences and Epidemiology. Handbook of Statistics. 2015;33:171-202. doi: 10.1016/B978-0-444-63492-4.00008-3.

18. Instituto Nacional de Estadística e Informática. Perú tiene una población de 32 millones 131 mil 400 habitantes al 30 de junio del presente año. 2020.2 Disponible http://m.inei.gob.pe/prensa/noticias/peru-tiene-una-poblacion-de-32millones-131-mil-400-habitantes-al-30-de-junio-del-presente-ano$\underline{11659 /}$

19. Lipsitch $M$, Cohen $T$, Cooper B, Robins JM, Ma S, James $L$, Gopalakrishna G, Chew SK, Tan CC, Samore MH, Fisman D, 
Murray M. Transmission dynamics and control of severe acute respiratory syndrome. Science. 2003 Jun 20;300(5627):1966-70. doi: 10.1126/science.1086616.

20. Guerra FM, Bolotin S, Lim G, Heffernan J, Deeks SL, Li Y, Crowcroft NS. The basic reproduction number (R0) of measles: a systematic review. Lancet Infect Dis. 2017 Dec;17(12):e420-e428. doi: 10.1016/S1473-3099(17)30307-9.

21. Anderson RM, Heesterbeek $H$, Klinkenberg $D$, Hollingsworth TD. How will country-based mitigation measures influence the course of the COVID-19 epidemic? Lancet. 2020 Mar 21;395(10228):931-934. doi: 10.1016/S0140-6736(20)30567-5.

22. Canals M, Cáceres $D$, Alvarado S, Canals A, Cattan PE. Modeling Chagas disease in Chile: From vector to congenital transmission. Biosystems. 2017 Jun-Jul;156-157:63-71. doi: 10.1016/j.biosystems. 2017.04.004.

23. Gozzer E, Canchihuamán F, Espinoza R. COVID-19 y la necesidad de actuar para mejorar las capacidades del Perú frente a las pandemias. Rev Peru Med Exp Salud Publica, 2020; 37(2). doi: 10.17843/rpmesp.2020.372.5410.

24. Munayco CV, Tariq A, Soto-Cabezas GG, Reyes, M. F, Valle A, Rojas-Mezarina L, Cabezas C, Loayza M, Chowell G. Peru COVID19 working group. Early transmission dynamics and control of COVID-19 in a southern hemisphere setting: Lima-Peru, February 29th-March 30th, 2020. Medrxiv. 2020 May 20. doi: 10.1101/2020.04.30.20077594.

25. Guan WJ, Ni ZY, Hu Y, Liang WH, Ou CQ, He JX, Liu L, Shan $H$, Lei CL, Hui DSC, Du B, Li LJ, Zeng G, Yuen KY, Chen RC, Tang $C L$, Wang $T$, Chen PY, Xiang J, Li SY, Wang JL, Liang ZJ, Peng YX, Wei L, Liu Y, Hu YH, Peng P, Wang JM, Liu JY, Chen Z, Li G, Zheng ZJ, Qiu SQ, Luo J, Ye CJ, Zhu SY, Zhong NS; China Medical Treatment Expert Group for Covid-19. Clinical Characteristics of Coronavirus Disease 2019 in China. N Engl J Med. 2020 Apr 30;382(18):1708-1720. doi: 10.1056/NEJMoa2002032.

26. Chan JF, Yuan S, Kok KH, To KK, Chu H, Yang J, Xing F, Liu J, Yip CC, Poon RW, Tsoi HW, Lo SK, Chan KH, Poon VK, Chan WM, Ip JD, Cai JP, Cheng VC, Chen H, Hui CK, Yuen KY. A familial cluster of pneumonia associated with the 2019 novel coronavirus indicating person-to-person transmission: a study of a family cluster. Lancet. 2020 Feb 15;395(10223):514-523. doi: 10.1016/S01406736(20)30154-9.

27. Chinazzi M, Davis JT, Ajelli M, Gioannini C, Litvinova M, Merler S, Pastore Y Piontti A, Mu K, Rossi L, Sun K, Viboud C, Xiong X, Yu $H$, Halloran ME, Longini IM Jr, Vespignani A. The effect of travel restrictions on the spread of the 2019 novel coronavirus (COVID-19) outbreak. Science. 2020 Apr 24;368(6489):395-400. doi: 10.1126/science.aba9757.

28. Fang $Y$, Nie $Y$, Penny $M$. Transmission dynamics of the COVID19 outbreak and effectiveness of government interventions: $A$ datadriven analysis. J Med Virol. 2020 Jun;92(6):645-659. doi: 10.1002/jmv. 25750 .

29. Wang $H$, Wang $Z$, Dong $Y$, Chang $R$, Xu C, Yu X, Zhang S, Tsamlag L, Shang $M$, Huang J, Wang $Y, X u G$, Shen $T$, Zhang $X$, Cai $Y$. Phase-adjusted estimation of the number of Coronavirus Disease 2019 cases in Wuhan, China. Cell Discov. 2020 Feb 24;6:10. doi: 10.1038/s41421-020-0148-0.

30. Aleta A, Moreno Y. Evaluation of the potential incidence of COVID-19 and effectiveness of containment measures in Spain: a data-driven approach. BMC Med. 2020 May 27;18(1):157. doi: 10.1186/s12916-020-01619-5. 